\title{
AN OVERVIEW OF RED-THERMOLUMINESCENCE (RTL) STUDIES ON HEATED QUARTZ AND RTL APPLICATION TO DOSIMETRY AND DATING
}

\author{
TETSUO HASHIMOTO \\ Faculty of Science, Niigata University, \\ Ikarashi-ninomachi, Niigata 950-2181, Japan
}

\begin{abstract}
The RTL-phenomena in quartz grains were initially reported and their properties and applications were developed by our group. Although RTL-measurements had the difficulty of overcoming high background counts due to blackbody radiation, the excellent dose linearity and hard-tobleaching nature of quartz RTL are suitable for accurate retrospective dosimetry and dating of burnt archaeological materials. In his paper, the discovery of RTL-phenomena is described. Subsequently, the construction and features of an automatic luminescence measuring system are mentioned together with innovative methods of decreasing the background level for the RTL measurement. Practical applications to dosimetry and dating are then described from the viewpoints of the preferable nature of RTL in comparison to other luminescence observations. Finally, some recent advances in RTL research are presented based on their emission mechanism correlated with impurity contents.
\end{abstract}

Keywords: OSL/RTL/IRSL phenomena, luminescence dating, archaeology.

\section{INTRODUCTION}

The red thermoluminescence (RTL) phenomena of quartz grains was discovered by our group from the TL photographic observations (Hashimoto et al., 1986a, b). The quartz RTL has been commonly found in volcanic ash layers and shown to give a broad emission band with a peak around $620 \mathrm{~nm}$ (Hashimoto et al., 1987). First application of RTL phenomena was carried out for the provenance search of dune sands on the basis of blue/red TL emission ratios using TL colour photographs (Hashimoto et al., 1989a; Ganzawa et al., 1997). The lifetimes of trapped electrons and holes related to RTL emissions were confirmed to be longer than $1 \mathrm{Ma}$ at ambient temperature which is long enough for archaeological dating (Hashimoto et al., 1993). The important utilization of the RTL as a dating tool for heated quartz was recognized, stemming from the good linearity of the dose response curve and the hard-to-bleach nature of quartz RTL by sunlight (Miallier et al., 1994a; Hashimoto et al., 1989b). On the basis of these advantages, a number of quartz RTL

Corresponding author: T. Hashimoto

e-mail: hashimoto-t@ginzado.ne.jp

ISSN 1897-1695 (online), 1733-8387 (print) (C) 2008 GADAM Centre, Institute of Physics, Silesian University of Technology.

All rights reserved. ages have been reported, including the dating of heated quartz associated with ancient volcanic events (Pilleyre $e t$ al., 1992; Miallier et al., 1991, 1994b, 1994c; Fattahi and Stokes, 2000a).

Quartz provenance studies have clarified the formation conditions of the blue TL (BTL) and RTL-quartz grains as being related to their different thermal histories; the former was assigned mainly due to plutonic rocks, whereas the latter originated commonly from volcanically-formed quartz and burnt archaeological materials (Hashimoto et al., 1991, 1996, 1998). Laboratory experiments revealed that the changes from BTL to RTL depend greatly on the impurity contents as well as on the cooling rate after thermal heating, and showed good agreement with the quartz RTL nature of burnt archaeological materials (Hashimoto et al., 1996 and 1998).

From a major development of apparatus, an automated luminescence measurement system made possible a new single aliquot regenerative dose (SAR) method especially for the optically stimulated luminescence (OSL) dating (Murray and Wintle, 2000). This SAR method has been also applied to the volcanic quartz older than 1.2 million years by the use of RTL measurement 
(Boetter-Jensen and Murray, 2001; Fattahi and Stokes, 2000a).

Two papers have appeared as reviews based on the number of publications that have, thus far, been concerned with the luminescence dating of volcanic and related sediments (Fattahi and Stokes, 2003; Stokes and Fattahi, 2003). In these reviews, particular attention is paid to the features of RTL and to practical applications of RTL dating using quartz fractions.

In the present paper, I describe the discovery of quartz RTL, followed by some advanced topics related to the RTL phenomena observed in our laboratory, such as the construction of a new luminescence measuring system, the effectiveness of RTL in archaeological dating and retrospective dosimetry, and an approach to understanding the mechanism of RTL production from impurity analysis.

\section{DISCOVERY OF RTL PHENOMENA FROM QUARTZ GRAINS OF DUNE SANDS}

As is well-known, the simplest technique for observing colour properties of luminescence phenomena, including thermoluminescence and phosphorescence from natural minerals, is a photographic method using either a colour film or an advanced digital camera. This technique offers not only colour information but also spatial information.

Fig. 1 (at the end of text) shows four TL colour photographs, in which the first one (a) shows a mixture of BTL- and RTL-quartz grains. The sample of quartz grains was separated from a sand dune on which our university is built. Prior to the mid-1980s, the TL colour from quartz was thought to consist only of a blue emission, so that the observation of red (RTL) emission was an attractive discovery in the luminescence field (Hashimoto et al., 1986a, 1986b).

To search for the cause of RTL quartz grains, the origins of the dune sands were traced. As a result, the RTL grains were found to be derived mainly from volcanic areas along the main river. In fact, photographs of the RTL of quartz grains from volcanic ash layers (Medeshima, Sendai, Japan) are shown in Fig. 1b. Almost all of volcanic quartz grains were later recognized as exhibiting RTL phenomena spread not only across volcanically active areas but also over the Earth surface (Aitken, 1998).

In the experimental approach to clarify the cause of RTL, Z-cut sliced samples from a crystal rock, Madagascan quartz of hydrothermal origin, were given a thermal annealing treatment, because volcanic quartz grains should be greatly affected by the heating and cooling process during and after the volcanic eruption. As a result, an as-received slice (Fig. 1c), which possesses only BTL property, changed partly into RTL-emitting regions (Fig. 1d) after being annealed for $100 \mathrm{hr}$ at $1000^{\circ} \mathrm{C}$ and then rapidly cooled. In the case of the regions with a clear RTL appearance, the applied temperature was confirmed to lie beyond the quartz to tridymite phase-transition temperature of around $870^{\circ} \mathrm{C}$ from (Hashimoto et al., 1996). Additionally, the changed regions were verified to contain many Al-impurities and much molecular water, whereas the unchanged (BTL) parts have lower impurity contents (see the section 5 for details; Hashimoto et al., 1997).

These results support the proposition that the elimination of BTL centre candidates will occur during thermal annealing treatment and subsequent radiation exposure at room temperature. That is, the thermal annealing effects play an important role in the RTL region that changed from BTL. Since there are a lot of volcanoes in Japan, it is reasonable to expect a mixture of BTL and RTL grains in the dune sands.

A more accurate TL spectrum has been observed using on-line spectrometer highly sensitive to the low luminescence from natural minerals (Rink et al., 1993; Hashimoto et al., 1997). Such kinds of TL spectral information, including that of RTL, were summarized from the viewpoint of relevance to luminescence dating by Krbetschek et al. (1997).

Fig. 2 shows a typical 3-dimensional (3-D) expression of the RTL spectrum. As can be seen in this figure, the RTL emission spectrum is confirmed to possess a maximum emission or peak around $630 \mathrm{~nm}$ and $360^{\circ} \mathrm{C}$, a result in close concordance with the 3-D spectra measured by Scholefild and Prescott (1999).

\section{CONSTRUCTION OF A NEW AUTOMATED LUMINESCENCE MEASUREMENT SYSTEM}

It is well-known that the reliable TL and OSL dating methods have been realized using a single aliquot regenerative dose (SAR) method which is superior to the multiple aliquots additive dose (MAAD) method from the aspect of requiring fewer sample aliquots (Fig. 3). In the SAR procedure, several repetitions are given of the luminescence measurement and dosing under a fixed geometrical condition by an artificial radiation source. Such experiments are commonly performed using an automated luminescence measuring apparatus equipped with

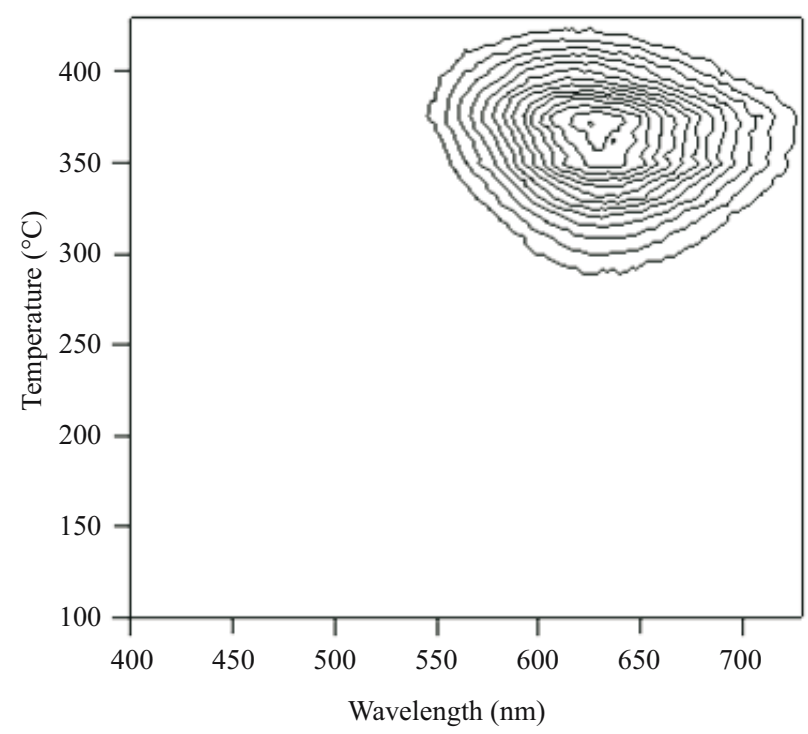

Fig. 2. Three-dimensional expression of RTL-spectrum for quartz extracted from an ancient pottery piece (Jōmon pottery, Okumiomote site, Niigata, Japan). 
a radioactive isotope ( $\mathrm{RI}$ ) source (amounting to 40-50 mCi), for example a Risoe TL-DA facility (Boetter-Jensen, 1997). However, the use of such RI sources requires radiation-shielding with material of heavy mass and limits the irradiation to a constant dose rate. Additionally, in Japan, such RI-irradiators are strictly restricted to certain locations regulated by law.

To overcome these difficulties, the author's group set out to design an automated luminescence-measuring assembly equipped with a small X-ray generator (Hashimoto et al., 2002a). Fig. 4 shows a conceptual view of a new automated luminescence measuring system that is applicable not only to BTL and OSL but also to RTL using the SAR method. The features of this assembly include a small $\mathrm{X}$-ray generator as an alternative radiation facility, a small surface heater, consisting of a cluster of ceramics heaters, in place of a nichrome plate heater, and

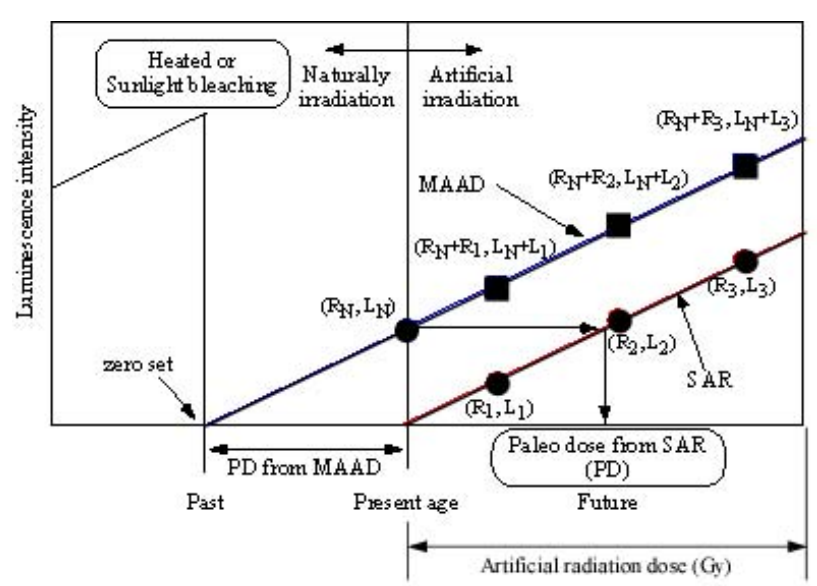

Fig. 3. Dose response curves for the single aliquot regenerative dose (SAR) and multiple aliquots additive dose (MAAD) methods of the evaluation of the equivalent dose for luminescence dating. a light guide, consisting of a glass core-rod, between the sample and a photo-multiplier tube (PMT), which is cooled to $-20^{\circ} \mathrm{C}$.

\section{Small X-ray generator}

The author's group were the first to propose the preferred use of a small X-ray generator (Varian VF-50J) instead of a ${ }^{90} \mathrm{Sr}-{ }^{90} \mathrm{Y} \beta$-source as the standard radiation source, because of the following advantages; (i) a close linearity between tube currents and X-ray dose-rates, (ii) a wide dynamic range of applicable dose rates, (iii) a highly uniform irradiation property to the sample area, and (iv) the possibility of controlling the applied tubevoltages and currents with a computer program (Hashimoto et al., 2002a, 2002b; Andersen et al., 2003; BoetterJensen et al., 2003).

Changes in the X-ray energy spectrum are shown in Fig. 5, in which the desired dose rates $(0.38 \mathrm{~Gy} / \mathrm{min} \sim$ $22.1 \mathrm{~Gy} / \mathrm{min}$ ) can be conveniently obtained by changing of the applied X-ray tube-voltages and currents. Additionally, an Al-absorber inserted between the sample pan and X-ray outlet greatly assist with the reproducible dosing of the mineral samples. The Al absorber effectively eliminates the low-energy X-ray components, which could affect heterogeneously the surface regions of quartz grains, but the higher energy X-rays pass through to produce homogeneous ionizing effects in the quartz grains. The use of an Al-absorber of $200 \mu \mathrm{m}$ thickness is recommended to evaluate equivalent doses with good reproducibility (Yawata et al., 2007). There appears no report whether such dose-attenuation corrections are necessary in the case of widespread beta-irradiation source.

\section{Ceramics heater assembly and light guide}

The contribution of black-body radiation to background counts is the most difficult problem to overcome

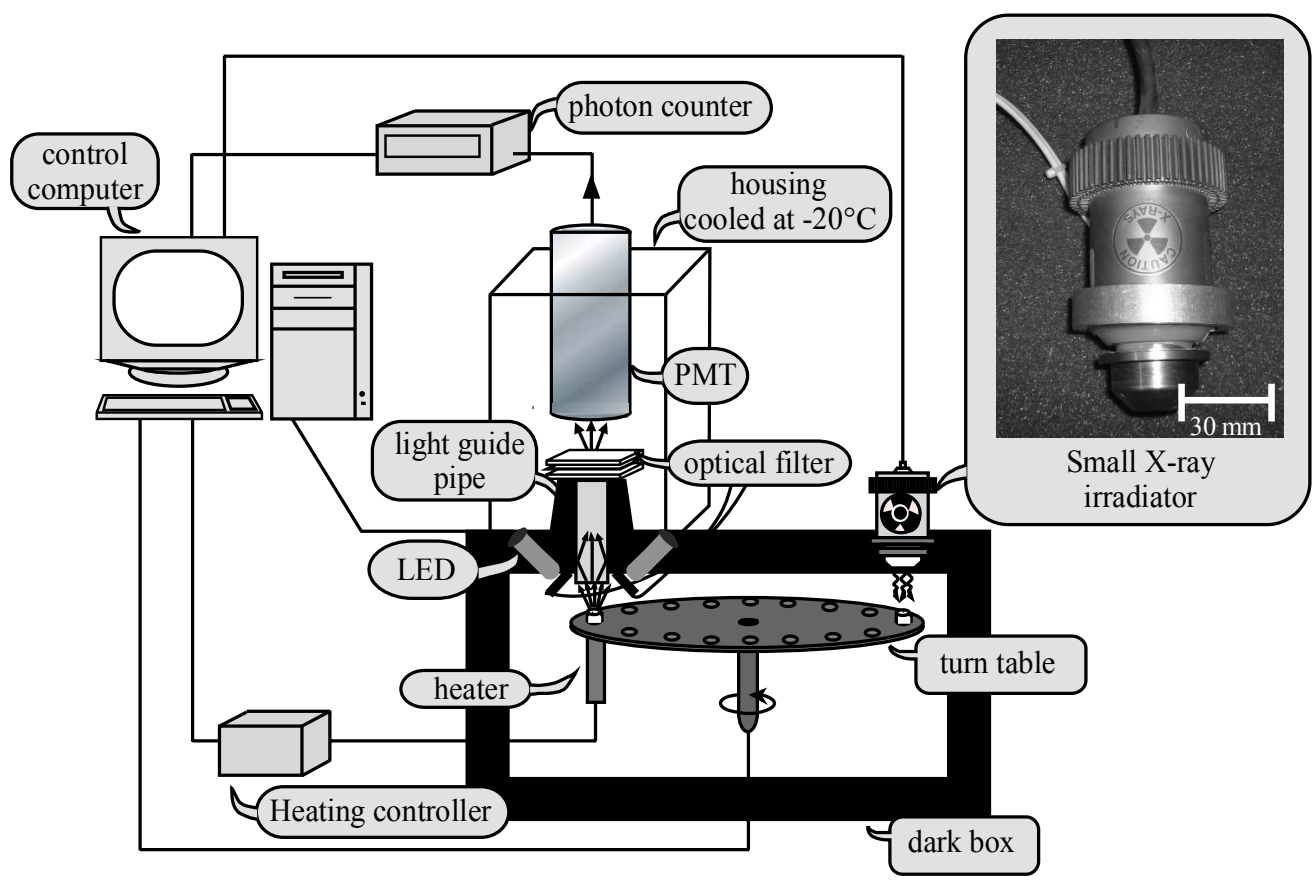

Fig. 4. Schematic diagram of an automated TL/OSL measurement system installed with a small $X$-ray generator. All measurement conditions were controlled by computer programs, including the operation of the $X$ ray generator. 
when measuring samples with extremely weak RTL, because the black-body radiation begins in the RTL wavelength regions when the heater and sample disk temperature rises above $250^{\circ} \mathrm{C}$. This problem has commonly been resolved by placing an appropriate selection of colour filter combinations in front of the PMT. Generally, a PMT with a multi-alkaline surface must be used in a cooled housing at $-20^{\circ} \mathrm{C}$ to keep such thermal noise as low as possible. The resultant long distance between the PMT surface and the sample pan mounted with luminescence material gives rise to a poor geometry for photoncollection.

The extent of this problem was reduced by using a glass light guide of core-rod type, which efficiently transferred luminescence without any loss and holding in thermal isolation of the parts between the heater and the sensitive surface of the PMT, which was cooled to $-20^{\circ} \mathrm{C}$; the reduced background count rate is seen in Fig. 6. As a result, the luminescence detection efficiency was enhanced by up to a factor of 40 compared with the same sample measured without the light guide.

For further reduction of the black-body radiation, the heater and sample pan area facing the PMT should be made as small as possible. To achieve this improvement, a special heater was designed; the heater part was composed of four ceramics heaters, with heating limited to the head parts, in a bronze capsule cover. Only the $10 \mathrm{~mm}$-diameter tabletop part of the bronze capsule directly faces the PMT (Hashimoto et al., 2002a, 2002b). In combination, these strategies reduced the total background to $2 \times 10^{4}$ counts per second in the $350-380^{\circ} \mathrm{C}$ region of the glow curve, corresponding to approximately one fifth of the best result achieved by Fattahi and Stokes (2000b).

A further major decrease of the black-body background was achieved by covering the sample pan with a biotite disk plate, leaving a hole for the luminescence sample. Fig. 7 shows the relation of hole diameter to the background value normalized to the value obtained without the biotite plate. Under these conditions, the thermal

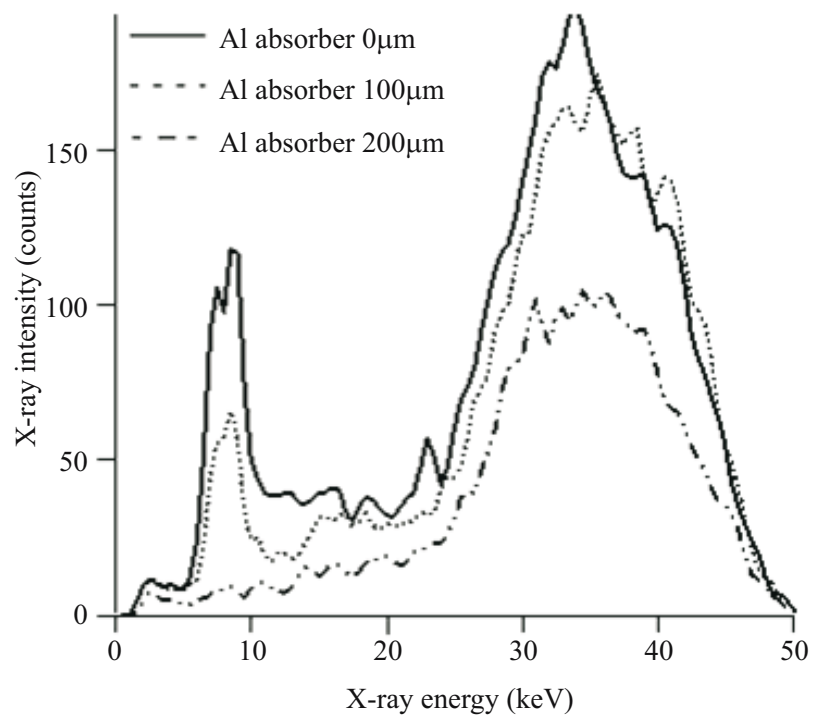

Fig. 5. Variations of $X$-ray energy spectra according to changes in thickness of $A$ l absorber. background decreased from $2 \times 10^{4}$ counts per second to 1,000 counts per second in the $350-380^{\circ} \mathrm{C}$ temperature range. Accordingly, the RTL detection limit was improved to about $100 \mathrm{cps}$, allowing the RTL measurement of individual quartz grains of about $0.15 \mathrm{~mm}$ in diameter (Yawata and Hashimoto, 2004, Ganzawa et al., 2005).

A recent advancement in our luminescence measurement system towards a portable assembly will appear in another paper (Takeuchi and Hashimoto, 2008).

\section{PRACTICAL USES OF RTL DATING AND DO- SIMETRY}

\section{Preferred RTL dating of burnt archaeological pottery}

Quartz and feldspar extracts from nine Jōmon pottery pieces, which were assumed to have been used 6,0003,500 years ago, were subjected to three kinds of lumi-

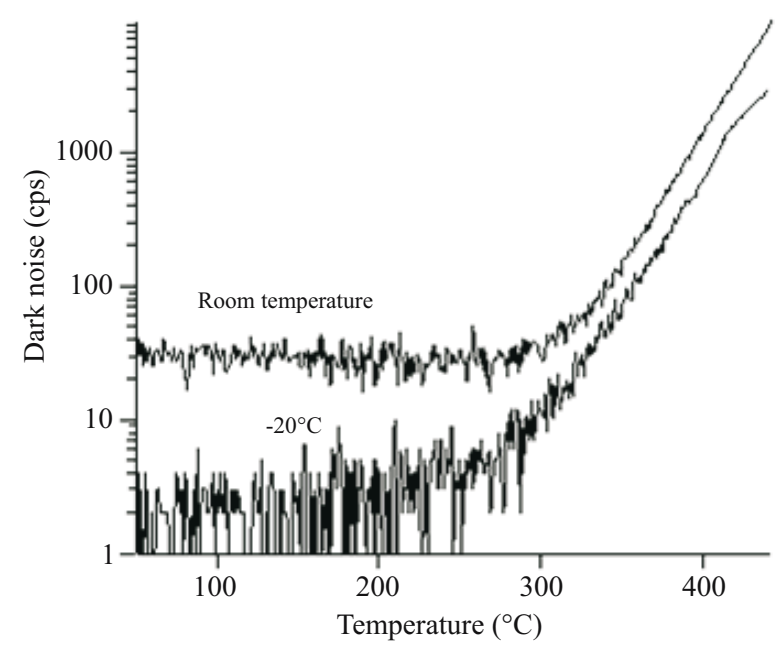

Fig. 6. Thermal background counts of a photo-multiplier tube (PMT: Hamamatsu Photonics, R-649S) during heating of a blank sample pan. For highly-sensitive RTL measurements, the background counts can be reduced by cooling the PMT to $-20^{\circ} \mathrm{C}$ and eliminating black-body radiation from the heater parts.

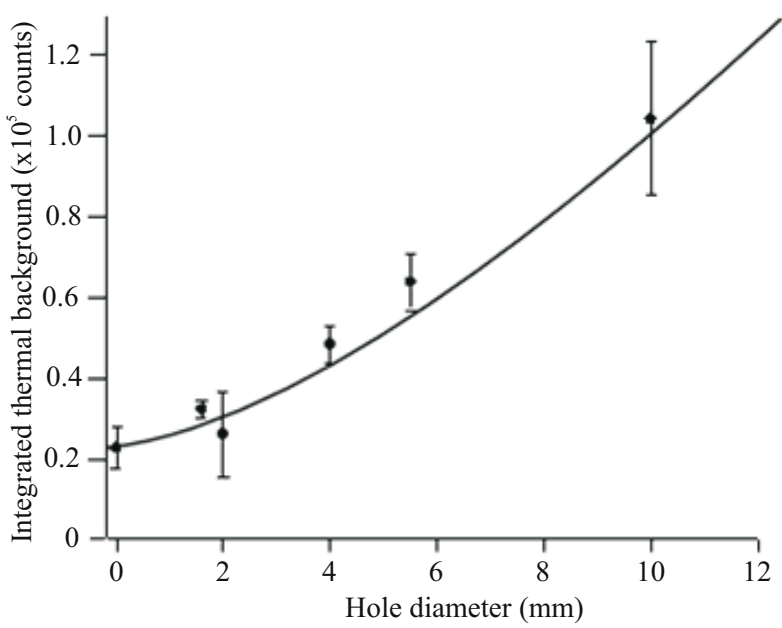

Fig. 7. Trend of declining background count as a function of the diameter of a hole, made in a biotite disk plate covering the top of the sample pan. The ordinate scale is normalized to the background count rate without the biotite plate. 
nescence measurements, involving RTL, OSL, and IRSL (infrared stimulated luminescence), using the automated luminescence measurement system (Hashimoto et al., 2005). The first two luminescence measurements were made on the quartz fraction, while the final one (IRSL) was made on feldspar one. All of the equivalent doses were estimated using the SAR protocol.

The results are summarized in Table 1. From this table, it is clear that the naturally accumulated doses measured using RTL are higher than the OSL and IRSL results. This is probably due to the relatively easily bleachable nature or instability of the luminescence centres and trapped electrons related to the OSL in quartz grains and the well-known anomalous fading effects on the IRSLrelated equivalent dose estimate for feldspar grains. The RTL ages, calculated using the equivalent doses and annual doses, which were estimated by $\gamma$-ray spectrometry, are close to the predicted archaeological ages, in contrast to the OSL and IRSL ages. Compared with the these latter, relatively unstable other luminescence phenomena, RTL-dating was found to be the most reliable dating method for quartz extracted from burnt archaeological materials. Recently, similar RTL dating was applied with satisfactory to younger burnt archaeological materials, such as roof tile pieces, used about 1,300 years ago (Hashimoto and Tamaki, 2007).

\section{RTL dosimetry of atomic bomb exposed roof tiles}

Several kinds of radiation-induced luminescence measuring methods, have been applied to retrospective dosimetry of roof tiles exposed to atomic bomb explosions at the Hiroshima and Nagasaki epicentres (Hashimoto et al., 2006a). All experimentally evaluated doses are presented in Table 2. In this table, the RTL measurements of the quartz fraction again exhibited the highest accumulated (equivalent) doses after a storage period of about 60 years, these values agreeing with previous dose calculations and evaluations (Roesch, 1987). Excluding RTL measurement of quartz, the underestimation of accumulated all doses from the quartz and feldspar extracts are thought to reflect the unstable properties of the asso- ciated luminescence centres and/or, for feldspar, the wellknown anomalous fading effects (Hashimoto et al., 2007a).

\section{DEPENDENCE OF TL PROPERTY ON IMPU- RITY CONTENT}

\section{Effects of $\mathrm{Al}$ and $\mathrm{OH}$ impurities}

On a Z-cut slice of a natural quartz crystal, some colour centre images (CCIs) were compared with thermoluminescence colour images (TLCIs), together with the distribution of $\mathrm{Al}$ from electron microprobe analysis. Other distribution images such as $\mathrm{OH}$-groups (as well as molecular water and Li-dependent $\mathrm{OH}$ impurity-species) from infrared-spectrometry showed almost similar patterns to $\mathrm{Al}$ as seen in Fig. 8b (at the end of text), giving high $\mathrm{OH}$-groups to $\mathrm{Al}$ content sites (Hashimoto et al., 2003). As seen in Fig. 8, dense colour-centre parts (CC) corresponded to parts with lower impurity contents and intense BTL emissions, whereas the colourless parts (CL) corresponded to zones with higher $\mathrm{Al}$ - and $\mathrm{OH}$-groups (and molecular water) impurities and with weaker BTL. Two small slices of quartz, distinguishable as CL and CC

Table 2. Retrospective doses evaluated from various kinds of luminescence emissions using quartz and feldspar extracts from atomic bombexposed roof tiles. See (Hashimoto et al., 2006a) for details.

\begin{tabular}{|c|c|c|c|c|c|c|}
\hline \multicolumn{2}{|c|}{ Sample } & RTL (Gy) & & BTL (Gy) & \multicolumn{2}{|c|}{ OSL (Gy) } \\
\hline Hiroshima & & $47 \pm 4$ & & $42 \pm 3$ & \multirow{2}{*}{\multicolumn{2}{|c|}{$\begin{array}{c}28 \pm 3 \\
8.2 \pm 1.1 \\
\end{array}$}} \\
\hline Nagasaki & & $11 \pm 2$ & & $6.7 \pm 1.5$ & & \\
\hline \multicolumn{7}{|c|}{ (b) feldspar grains ${ }^{*}$} \\
\hline Sample & $\begin{array}{c}\text { far-RTL } \\
(G y)\end{array}$ & $\begin{array}{l}\text { RTL } \\
\text { (Gy) }\end{array}$ & $\begin{array}{l}\text { GTL } \\
\text { (Gy) }\end{array}$ & $\begin{array}{l}\text { BTL } \\
\text { (Gy) }\end{array}$ & $\begin{array}{l}\text { UVTL } \\
\text { (Gy) }\end{array}$ & $\begin{array}{l}\text { IRSL } \\
\text { (Gy) }\end{array}$ \\
\hline Hiroshima & $35 \pm 3$ & $29 \pm 2$ & $23 \pm 3$ & $24 \pm 3$ & $22 \pm 5$ & $17 \pm 2$ \\
\hline Nagasaki & $8.4 \pm 1.3$ & $8.0 \pm 0.9$ & $6.7 \pm 0.9$ & $6.3 \pm 1.5$ & $5.7 \pm 1.7$ & $6.0 \pm 0.7$ \\
\hline
\end{tabular}

"The detection windows in wavelength: far-RTL $(700-750 \mathrm{~nm}), \mathrm{RTL}$ $(600-700 \mathrm{~nm})$, GTL $(500-600 \mathrm{~nm})$, BTL $(400-500 \mathrm{~nm})$, UVTL $(300-400$ $\mathrm{nm})$ and IRSL (350-600 nm).

Table 1. Equivalent doses (naturally accumulated doses) from RTL and OSL of quartz extracts and from IRSL of feldspar extracts and their luminescence ages. Burnt archaeological pottery pieces (Jömon-pottery excavated from Okumiomote ruin site, Niigata, Japan) were used for this intercomparison experiments.

\begin{tabular}{|c|c|c|c|c|c|c|c|c|}
\hline \multirow{2}{*}{$\begin{array}{l}\text { Pottery } \\
\text { pieces }\end{array}$} & \multicolumn{3}{|c|}{ Equivalent doses (Gy)* } & \multirow{2}{*}{$\begin{array}{c}\text { Annual dose } \\
\text { (mGyla) }\end{array}$} & \multicolumn{3}{|c|}{ Luminescence ages $\left.\left(a^{*}\right)\right)$} & \multirow{2}{*}{$\begin{array}{c}\text { Predicted } \\
\text { age (a) }\end{array}$} \\
\hline & RTL & OSL & IRSL & & RTL & OSL & IRSL & \\
\hline$\overline{A-5}$ & $13.1 \pm 4$ & $11.3 \pm 2$ & $0.7 \pm 0.2$ & $3.47 \pm 0.11$ & $3800 \pm 1150$ & $3300 \pm 600$ & $190 \pm 70$ & 4000 \\
\hline B-8 & $11.6 \pm 3.3$ & $5.6 \pm 2.2$ & n.d. & $2.17 \pm 0.1$ & $5300 \pm 1520$ & $2600 \pm 1000$ & n.d. & 6000 \\
\hline$B-10$ & $14.8 \pm 3.5$ & $6.3 \pm 0.8$ & $0.2 \pm 0.1$ & $2.18 \pm 0.09$ & $6800 \pm 1640$ & $2900 \pm 380$ & $100 \pm 40$ & 6000 \\
\hline B-12 & $26.0 \pm 2.2$ & n.d. & $3.9 \pm 0.1$ & $3.69 \pm 0.12$ & $7000 \pm 630$ & n.d. & $1100 \pm 40$ & 6000 \\
\hline$A-8$ & 17.3 & 15.2 & 0.92 & $3.27 \pm 0.11$ & $5300 \pm 180$ & $4600 \pm 160$ & $280 \pm 10$ & 5000 \\
\hline$A-14$ & $14.7 \pm 2.1$ & n.d. & $2.8 \pm 0.4$ & $3.17 \pm 0.11$ & $4600 \pm 670$ & n.d. & $880 \pm 140$ & 3500 \\
\hline$A-18$ & $15.0 \pm 2.4$ & $6.9 \pm 2$ & $2.0 \pm 0.2$ & $3.44 \pm 0.11$ & $4400 \pm 710$ & $2000 \pm 590$ & $570 \pm 60$ & 5000 \\
\hline A-23 & $12.2 \pm 1$ & $10.5 \pm 2.5$ & 0.13 & $3.01 \pm 0.11$ & $7500 \pm 1000$ & $3900 \pm 330$ & $2400 \pm 550$ & 6000 \\
\hline B-18 & $22.7 \pm 2.9$ & $11.8 \pm 0.9$ & $7.1 \pm 1.7$ & $3.17 \pm 0.13$ & $3800 \pm 360$ & $3300 \pm 800$ & $40 \pm 1$ & 3500 \\
\hline
\end{tabular}

${ }^{*}$ All ages refer to the year $A D 2,000$

*Errors are standard deviations from three measurements for each sample, except one measurement of A-8. 
parts, were examined to assess the changes in glow curve shape changes from liquid-nitrogen temperature to room temperature. The CL parts had more intense BTL glow curves below $-100^{\circ} \mathrm{C}$, while the $\mathrm{CC}$ parts gave apparently weaker BTL glow curves in the same low temperature regions. A thermal annealing treatment of $1050^{\circ} \mathrm{C}$ for $30 \mathrm{hr}$ resulted in a major decrease in the CL parts of the BTL intensity below $-100^{\circ} \mathrm{C}$, due to the expulsion of $\mathrm{OH}-$ groups and molecular water from the quartz grains. The results are shown in Fig. 9, in which Al hole centre and hydrogen radical intensities were measured by electron spin resonance (ESR) spectrometry. From these results, it has been suggested that hydrogen radicals and related reactive species as radiolysis products from $\mathrm{OH}$-groups and molecular water could erase $\mathrm{Al}$ hole centres, $\left(\mathrm{AlO}_{4} \mathrm{~h}^{+}\right)^{0}$, which acts as BTL centres above $-150^{\circ} \mathrm{C}$ (Hashimoto et al., 2003, 2006b). Similar results have been obtained from the growth zones, + and $-\mathrm{X}$ zones, of synthetic quartz samples (Tajika and Hashimoto, 2006).

On the basis of these results, hydrogen radicals and other radicals related to $\mathrm{OH}$ or water molecule impurities have been suggested as the cause of elimination of BTLcentres and that the BTL sensitivity of quartz at environmental temperatures is influenced not only by Alimpurities, but also by $\mathrm{OH}$ groups and other types of centre.

\section{RTL properties dependent on Al impurity contents}

To explain luminescence sensitivity variations and TL colour (RTL or BTL), 32 natural and synthetic quartz samples were analyzed for seven types of impurity content and for $\alpha-\beta$ phase transition temperatures (Yawata et al., 2006). Higher Al impurity content causes the inversion temperature to fall below the value of $573^{\circ} \mathrm{C} \mathrm{ob-}$ tained for pure synthetic quartz. The separation of RTLand BTL-quartz was classified at a boundary temperature

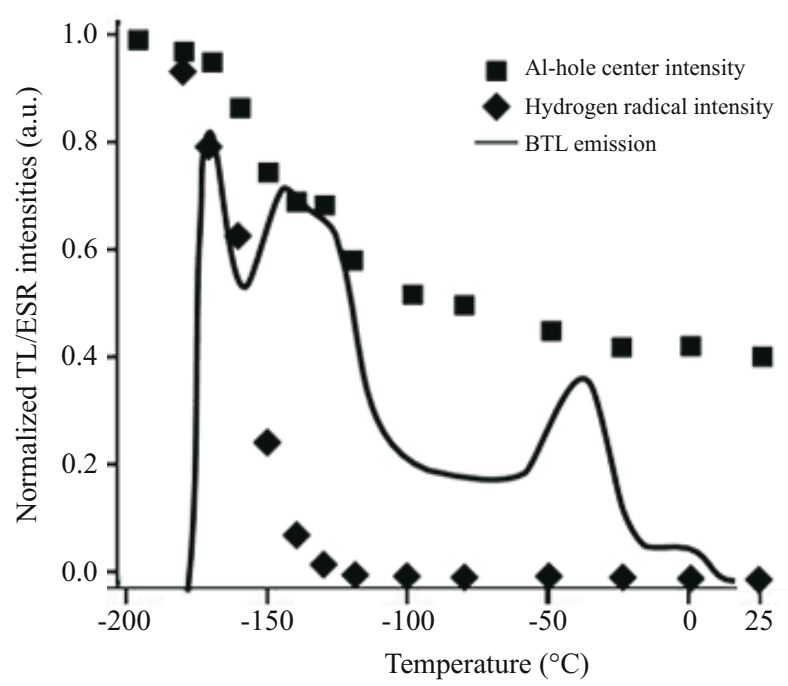

Fig. 9. BTL emission and isochronal annealing curves for Al hole centres and hydrogen radicals measured by ESR-spectrometry. The crushed quartz sample, being the colourless (CL) part in the colourcentre image as seen in Fig. 8a, was irradiated at liquid-nitrogen temperature, followed by the measurement shown (Hashimoto et al., 2006b). of $572{ }^{\circ} \mathrm{C}$, corresponding to Al-contents larger and smaller than $300 \mathrm{ppm}$, respectively. The RTL sensitivities increased in proportion to the approximate square of the Al content, strongly suggesting the co-operation of two closely spaced Al impurities as a RTL hole centre. According to these results, a model of RTL luminescence centres was proposed (Fig. 10), in which two nearby Al impurity atom substitutions give rise to the formation of RTL centres by bonding additional oxygen atoms or loss of an oxygen atom. This results in peroxy radicals or nonbonding oxygen centres, respectively, as tentative RTL centre candidates (Fattahi and Stokes, 2003; Hashimoto et al., 2007b).

\section{CONCLUSIONS}

Recent advances in spectrometric technique for detection of extremely weak luminescence emissions have allowed us to make accurate luminescence measurements. Additionally, reliable luminescence observations have been realized with a newly developed automated system of luminescence measurement together with the use of a SAR protocol that greatly suppresses the background level. In practical terms, the use of RTL from quartz extracts of burnt materials is preferred for both applications to dosimetry and luminescence dating applications.

To avoid a high background in ordinary RTL glow curve measurements, the isothermal-stimulation of red luminescence from quartz - measuring the RTL quartz at a lower constant temperature than the occurrence of black-body radiation - has recently been proposed and tested as an alternative RTL dating approach (Lai and Murray, 2006). Since a light-sensitive RTL portion was principally employed for such RTL dating, some fundamental and applied studies, involving light-sensitive and isothermally stimulated red emissions, have been carried out (Westaway and Roberts, 2006; Fattahi and Stokes, 2005; Miallier et al., 2006). Additionally, the far-red TL measurement of feldspar has investigated for possible applications to archaeological and sediment dating (Stokes and Fattahi, 2003; Hashimoto et al., 2007a), but space limitations prevent their discussion here.

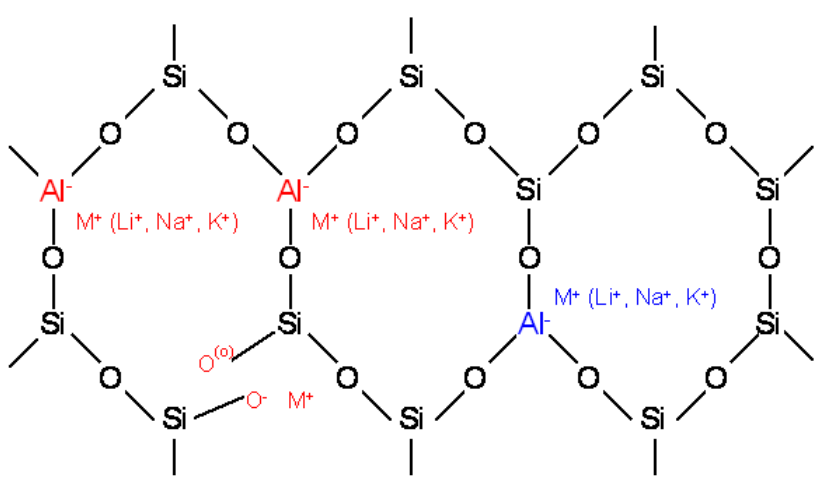

Fig. 10. RTL-centre model deduced from dependency of RTL on the square of the Al-impurity concentration in specimens of volcanic quartz. The strong distortion close to two Al substitution sites causes the rupture of the neighbouring Si-O-Si bond, where a RTL centre is formed. 


\section{ACKNOWLEDGEMENTS}

The authors would like to express his appreciation to Professor R. G. Roberts, University of Wollongong, Australia, Drs. T. Yawata and A. Takauchi, Graduated School of Science and Technology, Niigata University, for reading this manuscript and giving many corrections and valuable comments. This work was partly supported by a grant-in-aid for Fundamental Research from the Ministry of Education, Culture, and Sport, Japan (No. 17500691).

\section{REFERENCES}

Aitken MJ, 1998. An Introduction to Optical Dating. The Dating of Quaternary Sediments by the Use of Photon-stimulated Luminescence. Oxford, Oxford University Press: 267pp.

Andersen CE, Boetter-Jensen L and Murray AS, 2003. A mini X-ray generator as an alternative to a $90 \mathrm{Sr} / 90 \mathrm{Y}$ source in luminescence dating. Radiation Measurements 37(4-5): 557-561, DOI 10.1016/S1350-4487(03)00022-2.

Boetter-Jensen L, 1997. Luminescence techniques: instrumentation and methods. Radiation Measurements 27(5-6): 749-768, DOI 10.1016/S1350-4487(97)00206-0.

Boetter-Jensen L and Murray AS, 2001. Optically stimulated emission techniques in retrospective dosimetry. Radiation Physics and Chemistry 61(3-6): 181-190, DOI 10.1016/S0969-806X(01)002390 .

Boetter-Jensen L, Andersen CE, Duller GAT and Murray AS, 2003. Developments in radiation, stimulation and observation facilities in luminescence measurements. Radiation Measurements 37(4-5): 535-541, DOI 10.1016/S1350-4487(03)00020-9.

Fattahi M and Stokes S, 2000a. Extending the time range of luminescence dating using red TL (RTL) from volcanic quartz. Radiation Measurements 32(5-6): 479-485, DOI 10.1016/S13504487(00)00105-0.

Fattahi M and Stokes S, 2000b. Red thermoluminescence (RTL) in volcanic quartz: development of a high sensitivity detection system and some preliminary findings. Ancient TL 18: 35-43.

Fattahi M and Stokes S, 2003. Dating volcanic and related sediments by luminescence methods: a review. Earth-Science Reviews 62(3-4): 229-264, DOI 10.1016/S0012-8252(02)00159-9.

Fattahi M and Stokes S, 2005. Dating unheated quartz using a singlealiquot regenerative-dose red thermoluminescence protocol. Journal of Luminescence 115(1-2): 19-31, DOI 10.1016/j.jlumin.2005.01.012.

Ganzawa Y, Watanabe Y, Osanai F and Hashimoto T, 1997. TL colour images from quartzes of loess and tephra in China and Japan. $R a-$ diation Measurements 27(2): 383-388, DOI 10.1016/S13504487(96)00129-1.

Ganzawa Y, Furukawa H, Hashimoto T, Sanzelle S, Miallier D and Pilleyre T, 2005. Single grains dating of volcanic quartz from pyroclastic flows using red TL. Radiation Measurements 39(5): 479487, DOI 10.1016/j.radmeas.2004.10.012.

Hashimoto T, Hayashi Y, Koyanagi A, Yokosaka K and Kimura K, 1986a. Red and blue colouration of thermoluminescence from natural quartz sands. Nuclear Tracks and Radiation Measurements 11(4-5): 229-235, DOI 10.1016/1359-0189(86)90039-7.

Hashimoto T, Koyanagi A, Yokosaka K, Hayashi Y and Sotobayashi T, 1986b. Thermoluminescence colour images from quartz of beach sand. Geochememical Journal 20(3): 111-118.

Hashimoto T, Yokosaka K and Habuki H, 1987. Emission properties of thermoluminescence from natural quartz - blue and red TL response to absorbed dose. Nuclear Tracks and Radiation Measurements 13(1): 57-66, DOI 10.1016/1359-0189(87)90008-2.

Hashimoto T, Yokosaka K, Habuki H and Hayashi Y, 1989a. Provenance search of dune sands using thermoluminescence colour images (TLCIs) from quartz grains. Nuclear Tracks and Radiatation Measurements 16(1): 3-10, DOI 10.1016/1359-0189(89)90003-4.

Hashimoto T, Habuki H, Tanabe I, Sakai T and Takahashi S, 1989b. Dating using red thermoluminescence from quartz grains in strata related to fossil bones of Elephas Naumanni. Chikyukagaku (Geochemistry) 23: 35-43 (in Japanese).

Hashimoto T, Sakai T, Shirai N, Sakaue S and Kojima M, 1991. Thermoluminescent spectrum changes of natural quartzes dependent on annealing treatment and aluminium contents. Analytical Sciences 7(5): 687-690, DOI 10.2116/analsci.7.687

Hashimoto T, Kojima M, Shirai N and Ichino M, 1993. Activation energies from blue- and red-thermoluminescence (TL) of quartz grains and mean lives of trapped electrons related to natural redTL. Nuclear Tracks and Radiation Measurements 21(2): 217-223, DOI 10.1016/1359-0189(93)90165-6.

Hashimoto T, Notoya S, Arimura T and Konishi M, 1996. Changes in luminescence colour images from quartz slices with thermal annealing treatments. Radiation Measurements 26(2): 233-242, DOI 10.1016/1350-4487(95)00300-2.

Hashimoto T, Katayama H, Sakaue H, Hase H, Arimura T and Ojima T, 1997. Dependence of some radiation-induced phenomena from natural quartz on hydroxyl-impurity contents. Radiation Measurements 27(2): 243-250, DOI 10.1016/S1350-4487(96)00115-1.

Hashimoto T, Yasuda K, Sato K, Sakaue H and Katayama H. 1998. Radiation-induced luminescence images and TL-property change with thermal annealing treatment on Japanese twin quartz. Radiation Measurements 29(5): 493-502, DOI 10.1016/S13504487(98)00065-1.

Hashimoto T, Nakagawa T, Hong DG and Takano M, 2002a. An automated system for both red/blue thermoluminescence and optically stimulated luminescence measurement. Journal of Nuclear Science and Technology 39(1): 108-109, DOI 10.3327/jnst.39.108.

Hashimoto T, Nakagawa T, Usuda H and Yawata T, 2002b. Development of an automated system equipped with a small X-ray irradiator for red/blue thermoluminescence measurement from natural minerals. BUNSEKI KAGAKU 51: 625-632 (in Japanese).

Hashimoto T, Yamaguchi T, Fujita H and Yanagawa Y, 2003. Comparison of infrared spectrometric characteristics of Al-OH impurities and thermoluminescence patterns in natural quartz slices at temperatures below $0{ }^{\circ} \mathrm{C}$. Radiation Measurements 37(4-5): 479-485, DOI 10.1016/S1350-4487(03)00061-1.

Hashimoto T, Yawata T and Takano M, 2005. Comparison of naturally accumulated radiation-doses between RTL, BTL, OSL, and IRSL using white minerals from burnt archaeological materials and usefulness of RTL-dating from quartz extracts. Geochemical Journal 39(3): 201-212.

Hashimoto T, Fujita H, Sakaue H, Nakata Y and Nomura S, 2006a. Comparison of accumulated doses in quartz and feldspar extracts from atomic bomb-exposed roof tiles using several luminescence methods. Radiation Measurements 41(7-8): 1015-1019, DOI 10.1016/j.radmeas.2006.06.002.

Hashimoto T, Yamaguchi T, Tajika Y, Takeuchi A and Yawata T, 2006b. Behavior of TL glow curves for natural quartz samples from liquid-nitrogen temperature up to room temperature. Radiation Measurements 41(6): 671-676, DOI 10.1016/j.radmeas.2006.04.011.

Hashimoto T and Tamaki M, 2007. Preferable red thermoluminescence dating using quartz extracts from archaeological roof-tiles in Heijokyo ruin and thermoluminescence sensitivity comparison between quartz and feldspar fractions. Radioisotopes 56: 47-56 (in Japanese).

Hashimoto T, Sakaue H, Takeuchi T and Mitamura N, 2007a. Thermoluminescence (TL)-glowcurves from some feldspars and stability of far-red TL from albite. Radioisotopes 56: 7-16.

Hashimoto T, Yamaguchi T and Yawata T, 2007b. Blue and red thermoluminescence of natural quartz in the temperature region from $196^{\circ} \mathrm{C}$ to $400^{\circ} \mathrm{C}$. Radiation Measurements $42(3)$ : $341-346$, DOI 10.1016/j.radmeas.2007.02.064.

Krbetschek MR, Goetze J, Dietrich A and Trautmann T, 1997. Spectral information from minerals relevant for luminescence dating. $R a$ diation Measurements 27(5-6): 653-748, DOI 10.1016/S13504487(97)00223-0.

Lai Z-P and Murray A, 2006. Red TL of quartz extracted from Chinese loess: Bleachability and saturation dose. Radiation Measurements 41(7-8): 836-840, DOI 10.1016/j.radmeas.2006.04.017.

Miallier D, Fain J, Montret M, Sanzelle S and Soumana S, 1991. Properties of the red TL peak of quartz relevant to thermoluminescence dating. Nuclear Tracks and Radiation Measurements 18(1-2): 8994, DOI 10.1016/1359-0189(91)90098-3.

Miallier D, Fain J, Montret M, Pilleyre T, Sanzelle S, and Soumana S, 1994a. Sun bleaching of the red TL of quartz: preliminary observations. Ancient TL 12: 1-4.

Miallier D, Fain J, Sanzelle S, Pilleyre T, Montre M, Soumana S and Falgueres C, 1994b. Attempts at dating pumice deposits around $580 \mathrm{ka}$ by use of red TL and ESR of xenolithic quartz inclusions. 
Radiation Measurements 23(2-3): 399-404, DOI 10.1016/13504487(94)90070-1.

Miallier D, Sanzelle S, Falgueres C, Fain J, Montret M, Pilleyre T, Soumana S, Laurent M, Camus G and De Goer de Herve A, 1994c. Intercomparison of red TL and ESR signals from heated quartz grains. Radiation Measurements 23(1): 143-153, DOI 10.1016/1350-4487(94)90031-0.

Miallier D, Sanzelle S, Pilleyre T and Bassinet C, 2006. Residual thermo- luminescence for sun-bleached quartz: Dependence on pre-exposure radiation dose. Quaternary Geochronology 1(4): 313-319, DOI 10.1016/j.quageo.2007.01.003.

Murray AS and Wintle AG, 2000. Luminescence dating of quartz using an improved single-aliquot regenerative-dose protocol. Radiation Measurements 32(1): 57-73, DOI 10.1016/S1350-4487(99)00253$\mathrm{X}$.

Pilleyre T, Montret M, Fain J, Miallier D and Sanzelle S, 1992. Attempts at dating ancient volcanoes using the red TL of quartz. Quaternary Science Reviews 11(1-2): 13-17, DOI 10.1016/02773791(92)90036-8.

Rink WJ, Rendell H, Marseglia EA, Luff BJ and Townsend PD, 1993. Thermoluminescence spectra of igneous quartz and hydrothermal vein quartz. Physics and Chemistry of Minerals 20(5): 353-361, DOI 10.1007/BF00215106.

Roesch WC, 1987. US-Japan Joint Reassessment of Atomic Bomb Radiation Dosimetry in Hiroshima and Nagasaki (DS86). Final report. Radiation Effects Research Foundation, vol. 1: 432 pp.

Scholefield RB and Prescott JR, 1999. The red thermoluminescence of quartz: 3-D spectral measurements. Radiation Measurements
30(1): 83-95, DOI 10.1016/S1350-4487(98)00094-8.

Stokes S and Fattahi M, 2003. Red emission luminescence from quartz and feldspar for dating applications: an overview. Radiation Measurements 37(4-5): $383-395, \quad$ DOI $\quad 10.1016 / \mathrm{S} 1350-$ 4487(03)00060-X.

Tajika Y and Hashimoto T, 2006. Correlation of bluethermoluminescence (BTL) properties with some impurities in synthetic quartz. Radiation Measurements 41(7-8): 809-812, DOI 10.1016/j.radmeas.2006.05.014.

Takeuchi T and Hashimoto T, 2008. Construction of a portable mini luminescence-measuring system installed with a handy X-ray generator. Geochronometria 30: 17-22, DOI 10.2478/v10003-0080012-y.

Westaway KE and Roberts RG, 2006. A dual-aliquot regenerative-dose protocol (DAP) for thermoluminescence (TL) dating of quartz sediments using the light-sensitive and isothermally stimulated red emissions. Quaternary Science Reviews 25(19-20): 2513-2528, DOI 10.1016/j.quascirev.2005.06.010.

Yawata T and Hashimoto T, 2004. Identification of the volcanic quartz origins from dune sand using a single-grain RTL measurement. Quaternary Science Reviews 23: 1183-1186.

Yawata T, Takeuchi T and Hashimoto T, 2006. Dependence of luminescence sensitivities of quartz on $\alpha-\beta$ phase inversion break temperatures. Radiation Measurements 41: 841-846.

Yawata T, Hashimoto T, Takeuchi T and Hong DG, 2007. Optical conditions of X-ray irradiation for accurate equivalent dose determination. Nuclear Instruments and Methods in Physics Research B258: 375-380.
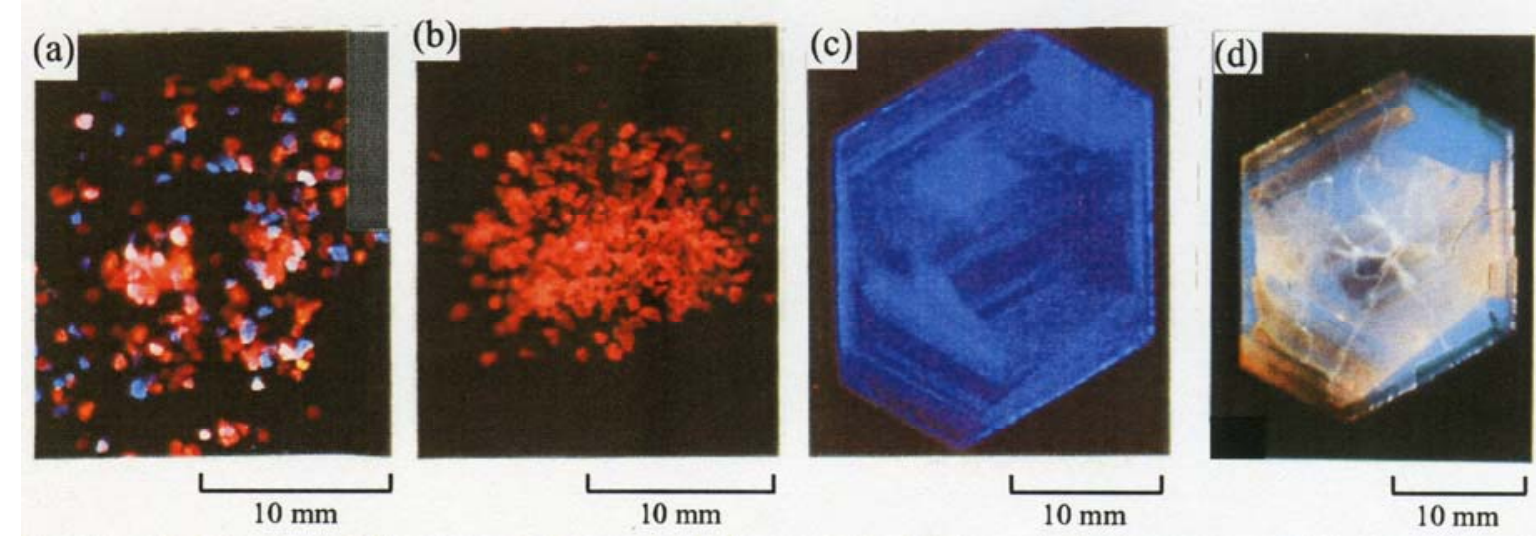

Fig. 1. Thermoluminescence colour images (TLCls). (a) Quartz extracts from Niigata dune sands; (b) quartz grains of volcanic origin from Tazawa lake (Akita, Japan); (c) original Z-cut slice of quartz of hydrothermal origin from Madagascar; (d) slice of quart - same as in (c) - after thermal annealing.
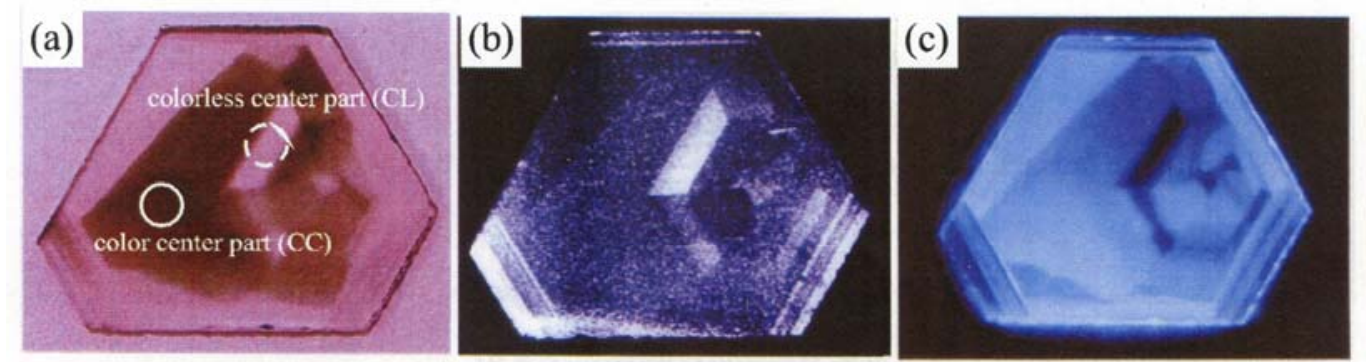

Fig. 8. Three 2-dimensional images from a Z-cut slice of a hydrothermal quartz crystal from Madagascar. (a); colour centre images after an irradiation of $20 \mathrm{kGy}$, (b); Al-impurity distribution from electron microprobe analysis, (c); $\mathrm{TLCl}$ from $200^{\circ} \mathrm{C}$ to $400^{\circ} \mathrm{C}$ for an irradiated slice. 